\title{
Partisan public health: how does political ideology influence support for COVID-19 related misinformation?
}

\author{
Nicholas Francis Havey ${ }^{1}$ (D) \\ Received: 6 July 2020 / Accepted: 11 October 2020 / Published online: 2 November 2020 \\ (c) Springer Nature Singapore Pte Ltd. 2020
}

\begin{abstract}
This study analyzes over 4000 tweets related to six misinformation topics about the COVID-19 pandemic: the use of hydroxychloroquine as treatment, the use of bleach as a preventative measure, Bill Gates intentionally causing the virus, the Chinese Communist Party intentionally causing the virus, and the Deep State causing the virus to ruin the economy and threaten President Trump's reelection chances. Across 5 of 6 topics (excluding bleach), conservatives dominate the discourse on Twitter. Conservatives are also more likely than their liberal peers to believe in and push conspiracy theories that the Chinese Communist Party, Bill Gates, and the Deep State are working in conjunction to infect the population and enact a surveillance state. Pandemic related misinformation has previously been associated with decreased adherence to public health recommendations and adverse health effects and evidence from the current pandemic indicates that adherence to public health recommendations is starkly partisan. This study suggests that the political and informational polarization further facilitated by social media platforms such as Twitter may have dire consequences for public health.
\end{abstract}

Keywords Sentiment analysis · Political polarization · COVID-19

\section{Introduction}

The emergence of the novel coronavirus (COVID-19) and its rapid spread have had demonstrable impacts on multiple sectors of society. Most prominently impacting public health and the global economy, COVID-19 has also revealed political divisions and provided evidence of a growing belief that science, and the empirical information it produces, is no longer trustworthy and is instead subject to partisan interpretation [1]. There is an increasing spread of information arguing that factors like 5G, Bill Gates, and the Chinese government are responsible for the pandemic [2,

Nicholas Francis Havey

nfh@g.ucla.edu

1 University of California, Los Angeles, Beverly Hills, CA 90210, USA 
3]. Experts are beginning to understand more about how beliefs in support of these conspiracy theories negatively impact public health efforts to control the spread of the virus; past research indicates that social media negatively impacts the uptake of preventative measures like social distancing and treatments like vaccines [4-6]. Identifying who is most susceptible to these conspiracy theories is thus necessary to combat both the spread of misinformation regarding COVID-19 and mitigate the harmful repercussions mistrust in public health recommendations facilitates. This paper finds that political conservatives predominate the misinformation discourse and support for conspiracies.

This study investigates how political ideology influences participation in the discourse surrounding support for or rejection of six COVID-19 conspiracy theories and misinformation topics: $5 \mathrm{G}$ activating the virus, Bill Gates using the virus as cover to implement a global surveillance project, the "Deep State" causing the virus, bleach and other household disinfectants as ingestible protection against the virus, hydroxychloroquine being a valid treatment for the virus (though its efficacy is widely disputed by medical researchers and there have been associated deaths and hospitalizations, the president has claimed he takes it preventatively), ${ }^{1}$ and the Chinese Communist party intentionally creating the virus.

\section{Theory}

The advent of the internet and social media, specifically social network platforms like Facebook and Twitter, has led to the "democratization" of information and the generation of an online and digitally mediated "public sphere" [7]. As more politically active people join social networks like Twitter, homophily and consensus-seeking lead to the transformation of the public sphere to "filter bubble" [8]. As others have shown, Twitter is increasingly more of an echo chamber than a public sphere [9] and political talk is highly partisan with a limited cross-ideological conversation $[10,11]$. The polarization of discourse, particularly around sensitive topics like the pandemic, can have dangerous repercussions.

Twitter in particular has also become a predominant space for populist messaging [12]. The democratization of information and the availability of social media platforms as nonhierarchical spaces for information distribution supports antielite populist messaging, particularly among extremist political groups [13]. In the American context, populist movements have cropped up behind Bernie Sanders and Donald Trump, though both people-centric movements have targeted decidedly different populations to criticize. Populist movements, particularly with nationalistic goals, lead people to consider the current system illegitimate and facilitate the support of often-untruthful demagogues [14]. Political leaders also often serve as informational quality heuristics for their followers [15], leading to increased consensusseeking and rejection of outgroup information [1]. Additionally, populist attacks on "mainstream" (outgroup / politically opposite) media have made it more difficult for

\footnotetext{
1 https://www.cnn.com/2020/05/18/politics/donald-trump-hydroxychloroquine-coronavirus/index.html.
} 
the layperson to discern what is true, particularly when the ideological group they identify with disagrees with something [16]. The proliferation of bots (automated accounts) and intentional spread of misinformation, or the amplification of a minority view through a fabricated, "grass-roots" movement (astroturfing) can create the false impression of widespread support for a topic, similarly impeding the identification of misinformation [17].

To the detriment of both political discourse and public health, populist surges have made truth increasingly irrelevant and accelerated the spread of misinformation [12, 18]. As Ribeiro, Calais, Almeida, and Meira Jr. put it, these days "everything I disagree with is \#FakeNews" [19]. The increasingly partisan assessment of information as fact or \#FakeNews is not entirely lopsided (Democrats participate in similar albeit less consensus seeking and homophily than their conservative counterparts), though Republicans are far more tolerant of misinformation from their leadership and critical of oppositional information than Democrats [20, 21]. This study thus seeks to investigate the following hypotheses:

Because conservatives are far more tolerant of misinformation from leadership $[20,21]$ :

$\mathrm{H}_{1}$ : Conservative Twitter users will tweet more than liberal Twitter users about all topics.

Because President Trump has been a vocal supporter of both Hydroxychloroquine and bleach as preventative measures or treatments for COVID-19:

$\mathrm{H}_{2}$ : For tweets about Hydroxychloroquine and bleach, conservative political ideology will positively correlate to support for (positive sentiment) each respective topic.

Because conservatives are far more likely to be critical of their opponents (here, Bill Gates, the Chinese Community Party, and the "Deep State", which is also regularly assumed or criticized for controlling certain parts of the world (like the 5G network) [19-21]:

$\mathrm{H}_{3}$ : For tweets about 5G, Bill Gates, the Chinese Communist Party causing the virus (CCVP), and the Deep State, conservative political ideology will negatively correlate to support for (positive sentiment) each respective topic.

As the successful identification of misinformation is important for the healthy functioning (literally, in the case of a pandemic) of a democratic society [22], combatting misinformation is crucial [23]. We know that misinformation is increasing and, in a public health crisis like a pandemic, identifying those most susceptible to it is imperative.

\section{Methodology}

\section{Overview}

Social media and the internet have expanded and altered the ways in which people communicate, spread and receive information, and engage in discourse within "unedited public spheres" [7]. The data Twitter and other social media platforms produce has been increasingly looked to as a way to observe social and political 
phenomena in situ, limiting the informational and disclosure-related concerns associated with traditional social science research methods like the interview and survey $[24,25]$. Social media, and Twitter data, in particular, has been used to document social movement activity [26-29], conversations and discourse around social justice issues [30-32], and general public sentiment towards specific topics [33-36].

Sentiment analysis, a method for gauging public sentiment or opinion on an issue, can be done with a variety of data types (interviews, open-ended survey responses, etc.). Tweets are increasingly becoming the topic of sentiment analyses, as they allow Twitter users the opportunity to relay short (280 characters or less) messages on a wide variety of topics to other users in their networks and later, through recirculation (retweets), those users' networks [37-41]. Sentiment analysis is primarily lexicon-based, removing "stop words" generally regarded as without sentiment (and, the, of, etc.) and assigning individual words affective scores based on their general use (hate is assigned a negative affective value) [39, 42, 43]. By assigning individual words in a singular tweet affective scores (positive, negative, neutral) and summing those overall scores (again, for a positive, negative, or neutral assessment), researchers engaging in sentiment analysis can parse large corpuses of data and reliably estimate how the public feels about a certain topic [44]. Individual words carry different sentiment intensities (hate being more negative than dislike, for example) and shorter strings, like tweets, are generally nudged in one direction or the other by individually strong word sentiments.

\section{Data collection and analytical approach}

To assess how political ideology influences discourse surrounding support for or rejection of various COVID-19 related misinformation topics (5G, Bill Gates, Deep State, Hydroxychloroquine, Bleach, and Chinese Communist Virus), I accessed the Twitter API (application programming interface) and requested the most recent tweets associated with each topic for the week of April 23rd to April 30th. To determine whether a tweet was associated with a topic, I searched for the keyword (5G, for example) and then used regular expressions to identify tweets containing topicrelevant words (COVID-19, coronavirus, virus, disease, pandemic). My initial sample consisted of a total of 5501 tweets with 357 hydroxychloroquine tweets, 877 Bill Gates tweets, 1849 bleach tweets, 353 Chinese Communist Virus tweets, 426 Deep State tweets, and $16395 \mathrm{G}$ tweets. After cleaning and organizing the data for each topic (I removed all tweets that did not contain the associated keywords or were not clearly related to the COVID-19 pandemic) and removing tweets by accounts with less than 50 followers or 50 friends and accounts that were younger than one month old (while users may have made Twitter accounts specifically to comment on the topics considered within this study, the newness of these accounts draws into question how real they are, as they may be being operated as bot accounts or sock-puppet accounts generated by existing users to amplify opinion on a particular topic), I retained a total of 4101 tweets with 276 hydroxychloroquine tweets, 661 Bill Gates tweets, 1323 bleach tweets, 287 Chinese Communist Virus tweets, 298 Deep State tweets, and $12565 \mathrm{G}$ tweets. 
After qualitatively reviewing a sample of tweets from each category to verify their relevance to the study (specifically reading to ensure that the regular expressions isolated tweets related to the pandemic), I separated each tweet by word and created unique word counts for each topic. Next, I compared each word in each topic corpus to the Bing Lexicon, a database with sentiment scores for individual words that was created using human-driven qualitative sentiment analysis [39, 42, $43,50]$ and assigned each word a sentiment score. The Bing Lexicon was developed by researchers interested in online opinion (their focus was specifically on customer reviews of products) and, after manually reviewing the entirety of the corpus and comparing what I found between this corpus and a portion of the corpus used to develop the Bing Lexicon, I felt that it was an appropriate lexicon to analyze these tweets with [50]. Specifically, the Bing lexicon allowed me to more narrowly analyze commonly abbreviated words used in short messages such as reviews and tweets that other lexicons did not include. It also offered a broader interpretation of strong opinion-based words like loathe, hate, and dislike, which helped to prevent all negative words being merged to similar values. While the Bing Lexicon was designed to assess online reviews, its utility for tweets, the majority of which commented on a user's particular stance towards one of the topics, is appropriate. After reviewing the words that were contributing most to tweet sentiment in each category, it became clear that Trump (assessed as positive within the lexicon) was skewing the data towards the positive; the word Trump was removed from subsequent analyses. Next, sentiment distributions for each keyword were calculated. Unique word indices, sentiment contribution indices, and sentiment distributions are displayed in the results section.

\section{The polarized public sphere}

As Twitter has rapidly become a source of breaking news, scraping Twitter for information regarding the public sentiment on political topics has been particularly relevant for both assessing public opinion and detecting misinformation in real-time [45]. Analysis of Twitter data has also revealed clear polarization surrounding the discussion of political topics like elections and debates [46]. Political discourse can also be extremely emotional, leading to further consensus seeking and polarization [47].

To assess how political ideology influenced discourse surrounding support for or rejection of the assorted COVID-19 conspiracy theories and misinformation topics (gauged by positive or negative sentiment scores, respectively), I used Barberá's Tweetscores R package to estimate a Twitter user's political ideology based on their association with elite users [48]. Barberá's Tweetscores packages utilize a Bayesian ideal point estimation approach to categorize users in comparison to a training set of politically elite users (Barack Obama, Hillary Clinton, Bernie Sanders, Glenn Beck, etc.) and assign them a political ideology score based on their association with Twitter elites. The package was validated on a set of millions of tweets comprised of millions of unique users discussing a variety of extremely political (elections) and extremely apolitical (sports) topics and has since been validated in the first author's 
other work and where the paper has been cited. As the tool has been well validated in the literature, successive validation was not necessary. I did, however, manually review a sample of highly partisan tweets to ensure they reflected the estimated ideology they were assigned. I found no discrepancies. Where association with elite users was unavailable [a user failed to follow any of the politically elite users in Barberá's training set (senators, politicians, partisan news personalities, etc.)], secondary estimation of their political ideology was achieved through analysis of a user's discursive habits (correspondence analysis-who they reply to, engage with, etc.). Users' estimated political ideologies fell on an existing scale ranging from between -2.7 and +2.7 with moderates occurring roughly between -0.4 and 0.4 , liberals between -0.4 and -2.7 , and conservatives between 0.4 and 2.7 [48]. Where users' political ideologies could not be estimated using either method, they were assigned a value of zero and subsequently removed from the analyses. Many of these user accounts had been deleted between the time of their tweet (4/23-4/30) and the full analyses (May). This suggests that many of these users were either bots or temporary accounts. While this significantly reduced the tweet corpus, removal of these transient users mitigated a potential skewing of the data with fabricated, fictitious, and temporary accounts (astroturfing). After assigning the tweets in the corpus a political ideology, each keyword's sentiment distribution was plotted against each associated tweet's political ideology.

\section{Limitations}

This study has several limitations. First, it is limited by its single time point and subsequent low amount of data when compared to larger (multimillion) Twitter studies; however, secondary review of tweets available for the same keywords has indicated that the discourse surrounding several of the conspiracy theories (hydroxychloroquine and bleach, for instance) has died down over the course of the pandemic and additional timepoints would have resulted in an insignificant increase to the overall data and would have also reflected a change over time in discussion to reflect other, more prominent topics (such as the Woodward book and other newly released information). I specifically re-accessed and reviewed additional for the topics on August 24th and October 4th. Manual review of the tweets accessed indicates that conversation on the topics has largely subsided while the conversation has surged on more recent issues surrounding COVID-19. In August, the discussion largely centered divergent opinions on the utility of masks. The October tweets were accessed shortly after the president was diagnosed with COVID-19, which predominated the discussion online. While the corpus is thus smaller than one would hope, it accurately and succinctly represents a snapshot of the pandemic and subsequently reflects the discussion at that point.

Second, while the data was plotted with respect to an account's number of followers, no network analysis was conducted, making it difficult to assess whether support for or rejection of the selected conspiracy theories was tightly clustered (a small but vocal minority advocating one way or another). Future research should consider exploring both content clusters and information spread. Third, sentiment 
analysis has trouble assessing the true sentimental value of satirical or sarcastic content ("I love how stupid the president is"), which is a limit of the automated content analyses. This was particularly a problem for some of the topics with more neutral trend lines, as qualitative manual review of many of the outliers (liberal support for drinking bleach) were sarcastic. Complete manual review of the corpus, however, did indicate the neutral themes for certain topics suggested below in the results. Similarly, depending on the phrasing of a sentence, a purely objective statement (study finds no benefit to patients) can be construed as having a positive sentiment by virtue of one strong word (here, benefit). This limits the immediate assessment of these tweets in particular, as purely objective tweets favored sentimental language. Future research should consider other approaches, such as structural topic modeling or other qualitative approaches to coding tweets, to more narrowly assess the content and sentiment of tweets while avoiding the pitfalls of sentiment analysis. Finally, as other researchers have shown, Twitter is not necessarily representative of the larger public and is most actively used by a politically active, educated, and a younger section of the population $[25,48]$.

\section{Results}

\section{Unique word counts}

Sentiment analysis relies upon words and their associated meaning. For each of the 6 categories of Tweets I reviewed, I created word indices demonstrating the top 15 words represented across tweets in each topic. These results are presented in Table 1. Across each topic, the most used word is obviously the keyword. Other top words indicate the overall tenor of conversations regarding each topic. For hydroxychloroquine, the most used words are mostly about President Trump, patients, the compound as a treatment, and FDA Trials. For Bill Gates, the most used words are squarely about the virus. For bleach, the most used words indicate how the president discussed bleach as a treatment for COVID-19-injection and drinking. The CCP Virus tweets similarly highlight the virus, its impact on the US, and the potential cancellation of the presidential primary. The Deep State tweets, which criticize an imagined second government (the Deep State) as creating the virus, mostly reference President Trump and Emerald Robinson, a journalist and White House press correspondent for Newsmax TV, a right-wing entertainment and news outfit, who has been vocally critical of other media outlets' coverage of COVID-19. Finally, the $5 \mathrm{G}$ tweets reflect a more international conversation about COVID-19, as reflected by several non-English characters.

\section{Sentiment contributors}

As sentiment analysis relies on the unique contributions to overall sentiment an individual word offers, review of the top sentiment contributors for each topic is crucial for accurately gauging what the majority of tweets about each topic are about. The 


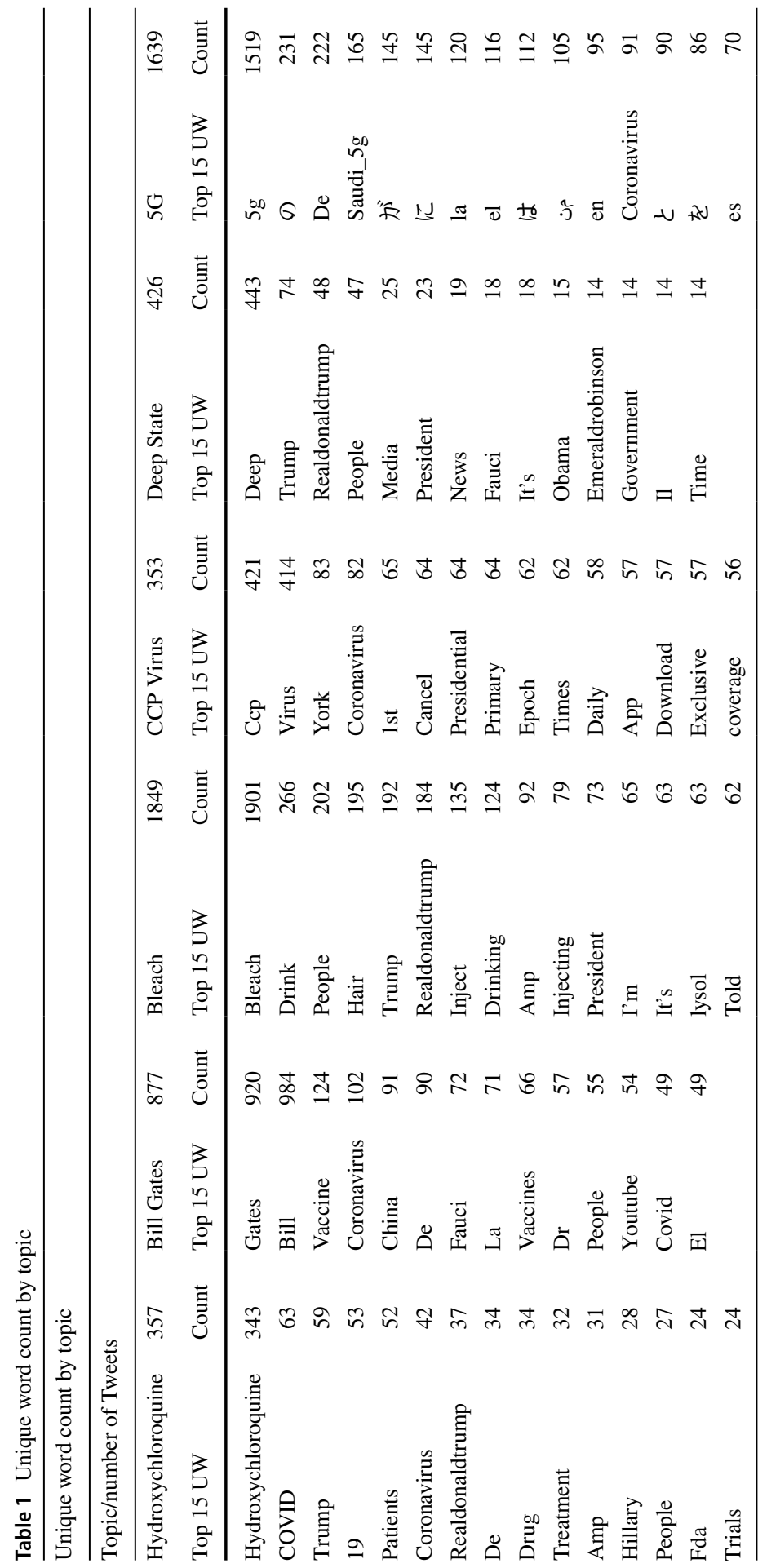




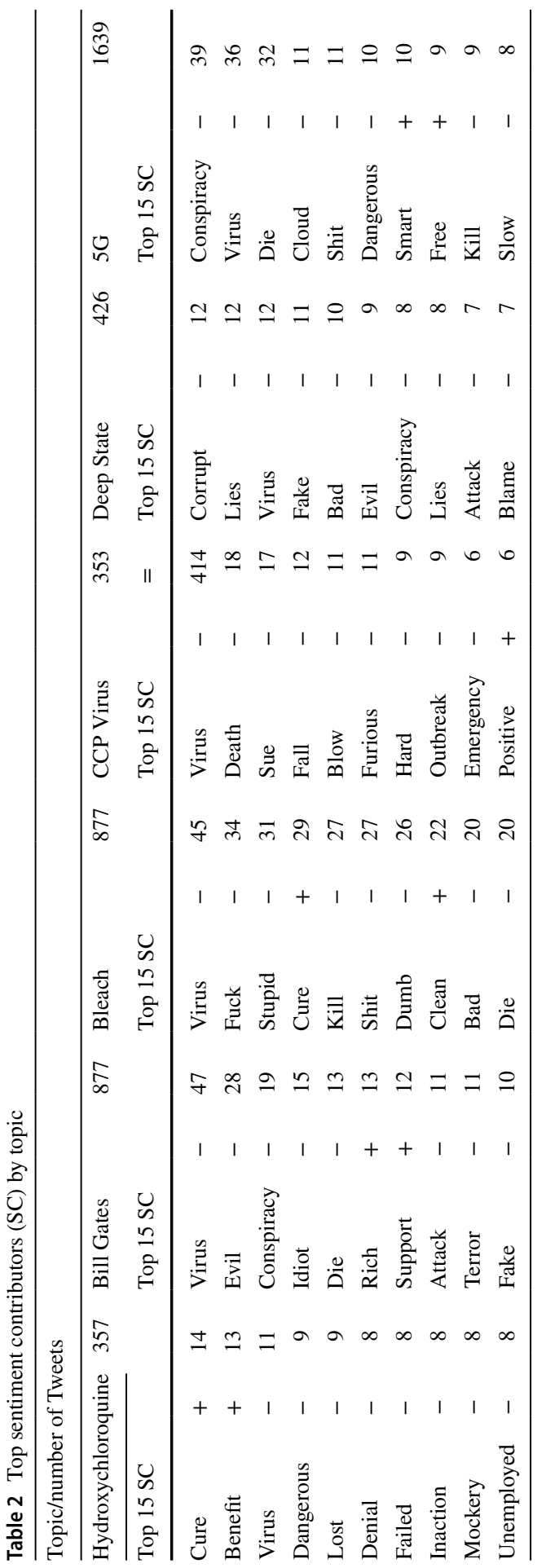




\section{Tweet Sentiment Percentages by Topic}

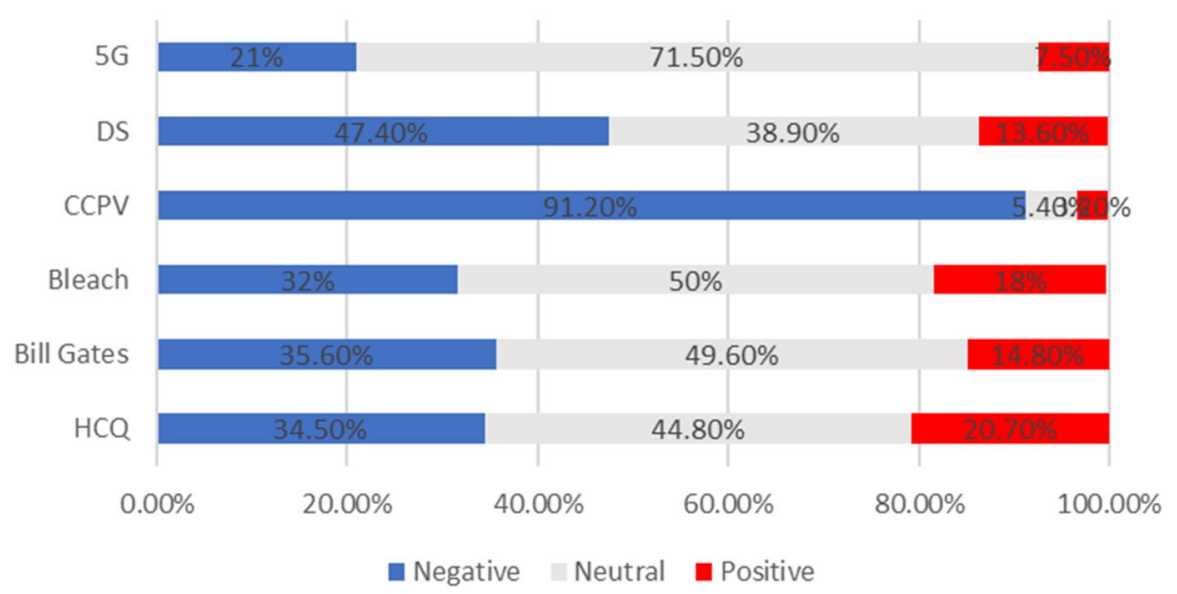

Fig. 1 Sentiment percentages by topic

top sentiment contributors for each topic are presented below, in Table 2. The top sentiment contributors for hydroxychloroquine tweets were specifically about the compound as a cure or benefit for the virus, but also noted the danger associated with words like dangerous, denial, failed, inaction, and mockery indicating the tone of the tweets. The top sentiment contributors for the Bill Gates tweets more squarely describe the topic of the conspiracy - that Bill Gates is waging an evil conspiracy to infect the world with a virus. The top sentiment contributors to the bleach tweets are similar to those for hydroxychloroquine, but are more unilaterally negative, with fuck, stupid, kill, shit, dumb, bad, and die making the top 15. The top sentiment contributors for tweets about the "CCP Virus", Deep State, and 5G mirror the contributors for the tweets about Bill Gates: they largely reference the COVID-19 pandemic as a result of corruption, lies, conspiracies, and a concerted effort to hurt the population (as evidenced by die, death, evil, etc.)

\section{Tweet sentiments by topic}

After calculating unique sentiments for each tweet in a topic's corpus, I calculated the percentage of each topic that was positively, neutrally, and negatively discussed. Results per topic are displayed in Fig. 1. Tweets about hydroxychloroquine, Bill Gates, and bleach were about half neutral (44.8, 49.6, and 50\%, respectively; neutral tweets are largely informative tweets that either reshare information or a link with minimal comment). Tweets about 5G were overwhelmingly neutral (71.5\%), and qualitative review of this tweet corpus indicates that most of these tweets were sharing information about $5 \mathrm{G}$ connectivity or sharing links to information debunking the idea that $5 \mathrm{G}$ was "triggering the virus." The nonneutral tweets for hydroxychloroquine, Bill Gates, bleach, and 5G were more negative than positive. Finally, tweets 
about the Deep State and the "Chinese Communist Party Virus" (CCPV) were predominantly negative ( $47.4 \%$ and $91.2 \%$, respectively).

\section{Tweet ideologies by topic}

After calculating political ideologies for the users in each topic corpus (where available), the percentage of tweets per topic that were tweeted by a liberal user, a conservative user, or a user with an incalculable ideological score were calculated. Results per topic are displayed in Fig. 2. For every topic except bleach, the largest group tweeting was conservative. For the more clear cut conservative topics (Bill Gates, CCPV, Deep State), conservative users were the overwhelming majority of discussants with 77.3, 90, and $85.6 \%$, respectively. These results partially and positively support $\mathrm{H}_{1}$, that conservatives tweet more than their liberal peers about the selected misinformation topics. With the exception of bleach, which was the largest corpus of tweets, conservatives discussed misinformation topics related to COVID19 in greater numbers than their liberal counterparts.

\section{Tweet sentiment by political ideology}

After calculating the sentiment and user's political ideology for each tweet in my corpus, I plotted tweet sentiment against political ideology to better understand how political ideology influenced participation in discussion about and support or rejection of my selected misinformation topics. To emphasize and identify tweets from users with large followings, tweets were resized by their user's follower count. Tweet sentiment ranges for each topic with a sentiment of 0 being roughly neutral (mostly informational tweets) and positive or negative sentiments corresponding to associated levels of feeling. On average, sentiment ranged from -8 to 8 with one outlier (a - 20 that was extremely negative). Political ideology similarly ranges across the topic, but liberal political ideology roughly begins around -0.4 and conservative political ideology around 0.4 . This is depicted graphically by a color gradient from blue (liberal) to red (conservative). Finally, several tweets from each topic (those with either large followings or an intense sentiment, or both) were selected to briefly illustrate the tone of each topic.

\section{Hydroxychloroquine}

I hypothesized $\left(\mathrm{H}_{2}\right)$ that tweets about hydroxychloroquine and bleach, which were roundly criticized in the liberal media but considered and defended by conservative media, would show a positive correlation between conservative political ideology and positive tweet sentiment. Figures 3 and 4 depict the relationship between tweet sentiment and political ideology for tweets specifically mentioning hydroxychloroquine and bleach. For hydroxychloroquine, the tweet representing the large purple dot with a sentiment of -2 was sent by @PalmerReport, reads "Donald Trump's hydroxychloroquine scandal just got even uglier https://t.co/HwVECBbsgU" and links to an article published by the Palmer Report strongly criticizing President 


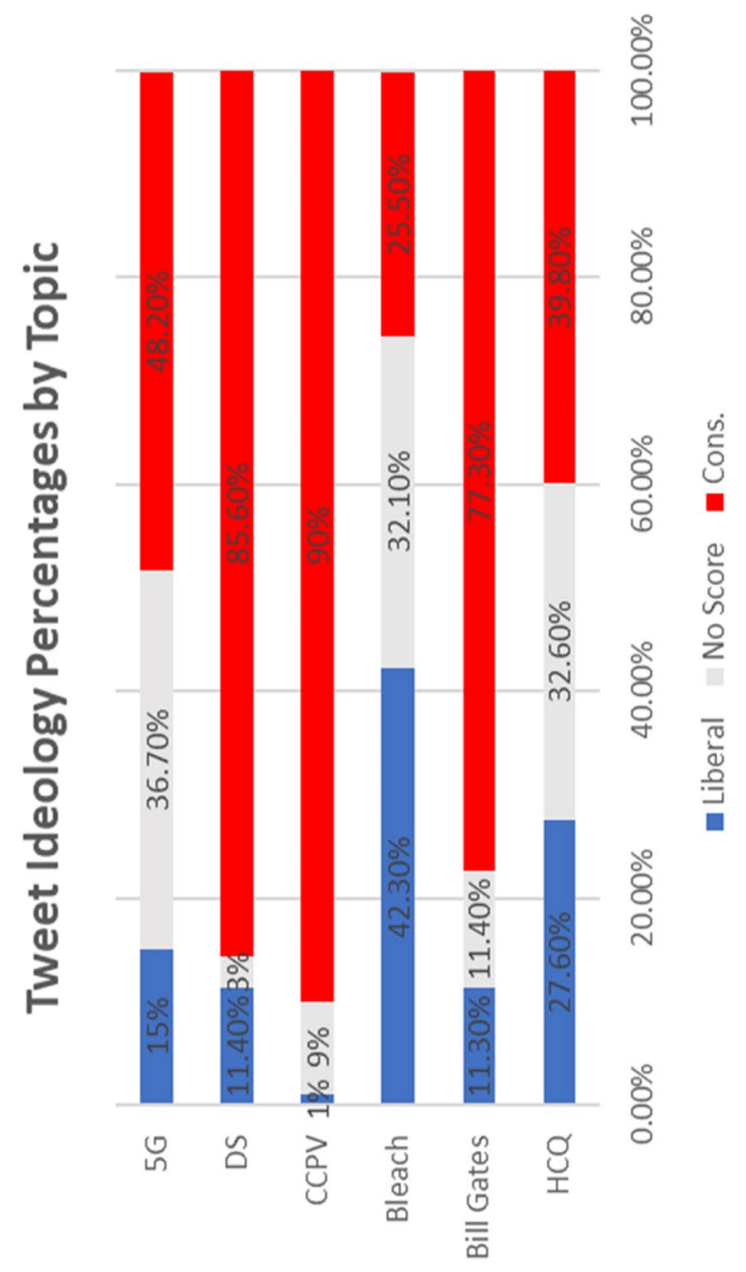

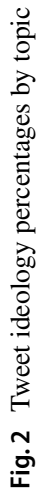




\section{Hydroxychloroquine Tweets}

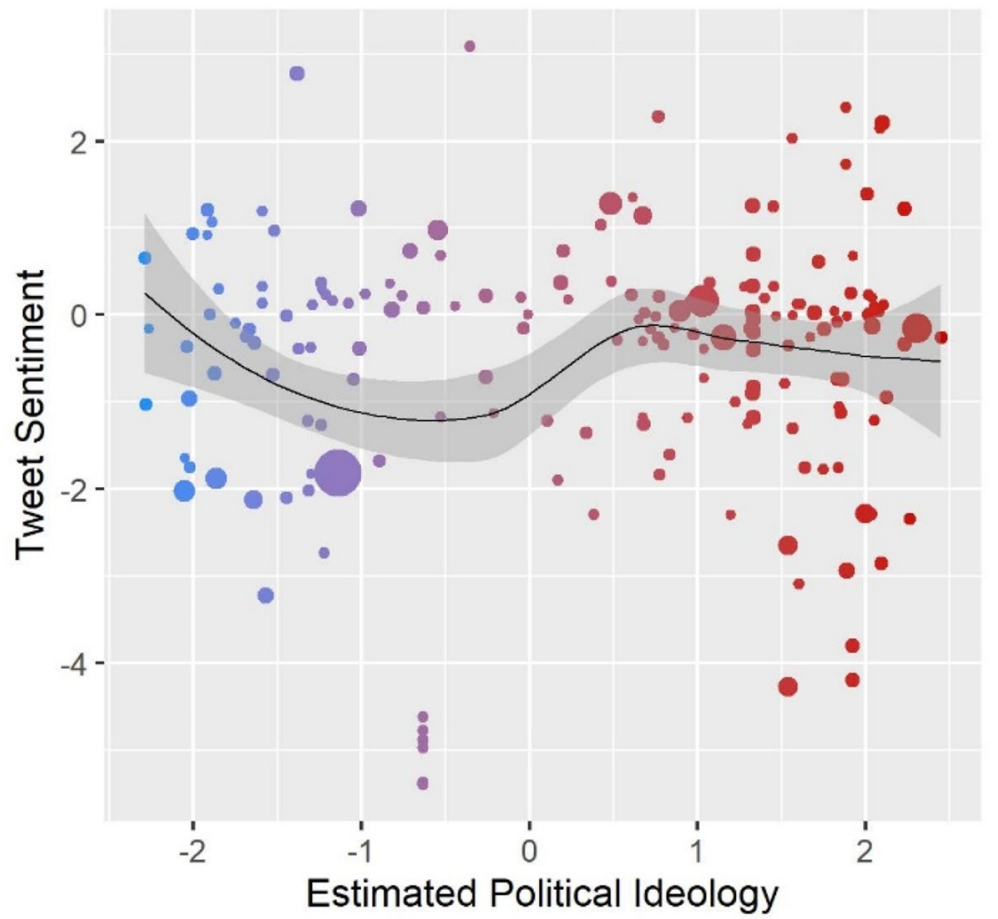

Fig. 3 Hydroxychloroquine Tweets

Trump's comments on hydroxychloroquine. On the other end of the political spectrum (1.92 estimated ideology), a negative tweet with a sentiment of -4 sent by @SkipStein reads "When the TRUTH finally comes out, lawyers will be so busy for years suing for damages, Constitutional Rights violations and wrongful death. States, MSM and corrupt doctors who Refused to administer hydroxychloroquine better lawyer up fast," both siding with the president and criticizing (and threatening) the mainstream media (MSM), states, and doctors for suppressing the "truth" about hydroxychloroquine. Similarly, a tweet sent by user @ tjnosweat (1.88 estimated ideology) reads "@ greggutfeld How did we learn about hydroxychloroquine, heat and UV sunlight and the fact that most states are ready to partially reopen? It wasn't from the task force experts, it was from President Trump. That's why he needs to speak at the briefings," whose positive view of the president aligns well with his political ideology and belies some level of nonchalance towards COVID19 (ready to partially reopen). While these individual tweets speak to a relationship between conservative political ideology and support for both the president and his miracle drug hydroxychloroquine, the relationship across the entire corpus does not show a positive relationship between positive sentiment towards hydroxychloroquine and conservative political ideology, partially refuting $\mathrm{H}_{2}$. Secondary qualitative 


\section{Bleach Tweets}

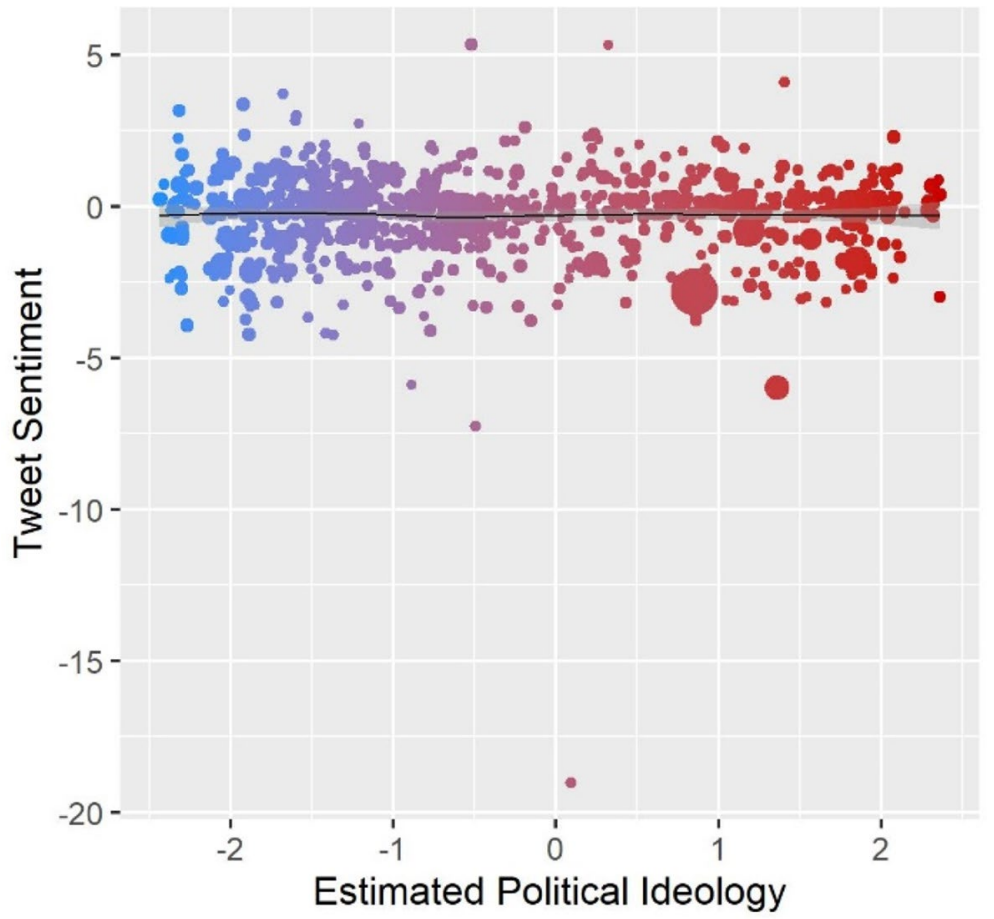

Fig. 4 Bleach Tweets

analysis of the positive and liberal tweets within the corpus (WTF: Australia continues trials with the Trump magic pudding drug hydroxychloroquine despite studies showing no benefit to coronavirus patients. \#covid19 \#AuspolSoCorrupt \#auspol https://t.co/5hCdpiWHS3, + 3 sentiment and - 1.38 estimated ideology; Australian hydroxychloroquine trials continue despite studies showing no benefit to coronavirus patients, by @MelissaLDavey https://t.co/xthIEHWilw, + 1 sentiment and - 1 estimated ideology) reveal largely objective and informative tweets about the Australian hydroxychloroquine studies. Words like benefit, magic, and continue strongly contributed to the sentiment without necessarily conveying support, a limitation of this analysis.

\section{Bleach}

I similarly hypothesized $\left(\mathrm{H}_{2}\right)$ that tweets about bleach would be negative from liberals and more supportive from conservatives. The trend line indicates no significant relationship between tweet sentiment and estimated political ideology for tweets about bleach, partially refuting $\mathrm{H}_{2}$. While there is no meaningful correlation between tweet sentiment and estimated political ideology when it comes to bleach, closer 
inspection of some of the wider-reaching tweets about bleach reveal strong condemnations of the President's application for bleach (injection, oral consumption). One tweet from user@1813Doncarlo (- 1.88 estimated ideology) reads "At least Brian Williams \& Don Lemon aren't stupid, moronic, idiotic, ignorant or deranged enough to advise \& encourage their viewers to drink bleach or inject disinfectant. https://t. co/mh0hhkY7mJ" - a reply to this tweet from President Trump "I must admit that Lyin' Brian Williams is, while dumber than hell, quite a bit smarter than Fake News @ CNN "anchorman” Don Lemon, the "dumbest man on television". Then you have Psycho Joe "What Ever Happened To Your Girlfriend?" Scarborough, another of the low I.Q. individuals!"- - is a prime indication of a negative response to the use of bleach for the treatment of COVID-19. While the previous tweet was clearly negative in tone, a lot of the "positive" tweets associated with bleach and sent from liberal users are evidently sarcastic, like this from user @jaedevereaux (- 1.60 estimated ideology): "Trump supporters are legally obligated to drink bleach by May 1, as decreed by their supreme leader, President Doland Trump." Finally, echoing my own concerns regarding the president's wanton disregard for facts, user @WisePaxCat $(-1.875)$ tweeted: "What if the president threatens to fire officials who refuse to echo his dangerous speculations about injecting bleach? https://t.co/h2QfnuWoB7 via@slate".

\section{G}

I hypothesized $\left(\mathrm{H}_{3}\right)$ that, for tweets about 5G, Bill Gates, the Deep State, and the Chinese Communist Party, there would be a negative correlation between conservative political ideology and positive sentiment (support) for each topic. Figures 5 and 6 depict the relationship between tweet sentiment and political ideology for tweets about 5G and Bill Gates, respectively. With respect to tweets about 5G, while conservatives dominate the conversation, there is not a clear trend, though there are far more negative tweets than positive tweets overall, thus partially supporting $\mathrm{H}_{3}$. One of the most negative tweets ( -5$)$, sent by user @EmilyCragg (1.60 estimated ideology), harshly criticizes California Governor Gavin Newsom and claims that $5 \mathrm{G}$ is one of the root causes of the pandemic: “@GavinNewsom He has no right to tell us where \& when we can go anywhere; we know this because the "virus" is not what it's cracked up to be; it's a composite between effects of $5 \mathrm{G}$, bad $\mathrm{O}_{2}$ saturation of air and various pulmonary diseases. He's a liar, a globalist intent on strife. https://t.co/ DPjTRvHv1D" (This was in response to an update on April 27th that stay-at-home orders would persist in California). The tweet representing the largest red dot (user @CNET, estimated ideology 0.6) was actively working to combat this sort of misinformation "There's no scientific evidence to suggest $5 \mathrm{G}$ has anything to do with coronavirus. Let's debunk some conspiracy theories. https://t.co/ypSf4NZnzL" and links to a video debunking popular conspiracy theories associated with 5G. Overall, discussion surrounding 5G was international, often only tangentially related to COVID-19 ("Are 5G towers bad bad? Cuz they put one right in front of my house and I'm scared Ik this shit raidiating off my phone somehow. I don't want cancer."), and frequently informational. 


\section{Five G Tweets}

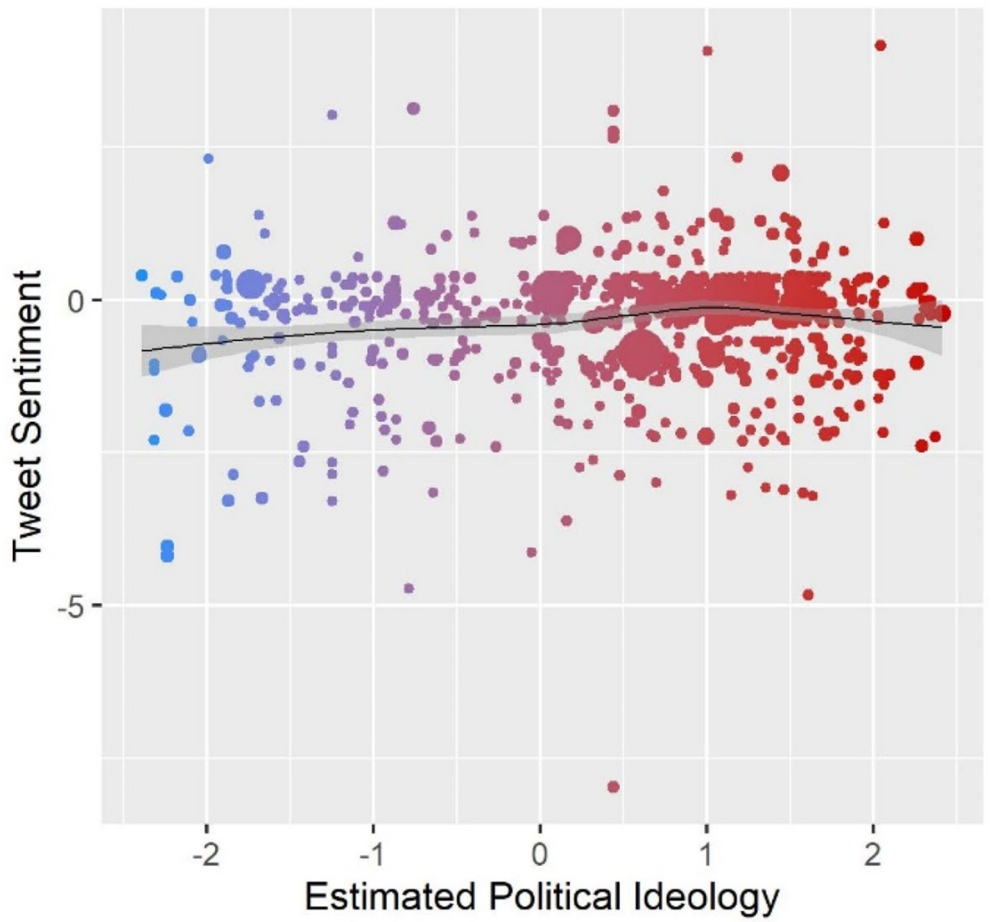

Fig. 5 G Tweets

\section{Bill Gates}

I similarly hypothesized that tweets about Bill Gates causing COVID-19 as a way to install a global surveillance state would be increasingly negative with relation to political ideology. While conservative Twitter users dominated the conversation around this conspiracy theory, average sentiment hovers just below 0 , indicating that the majority of tweets about this topic were purely informational or neutral in tone. The lack of a clear relationship thus partially refutes $\mathrm{H}_{3}$. Looking more closely at some of the tweets with larger reach, there is, however, evidence that some support this conspiracy theory on Twitter. User @ CRRJA5 (2.29 estimated ideology) tweets “@DonnaWR8 I'm NOT getting any vaccine made by Bill Gates" and user @CarlosCardetFL (1.7 estimated ideology) less subtly connects Bill Gates with the Chinese Communist Party: “@chenweihua Ever think that Bill Gates may have a vested interest in protecting the totalitarian dictatorship of Communist China and that this little rant of his may be a distraction from what truly happened in Wuhan?" User @ CamilleSeymour2 (2.13 estimated ideology) draws similar connections and calls upon right-wing talking head Candace Owens as proof: “@RealSaavedra Candace Owens Speaking Truth and common 


\section{Bill Gates Tweets}

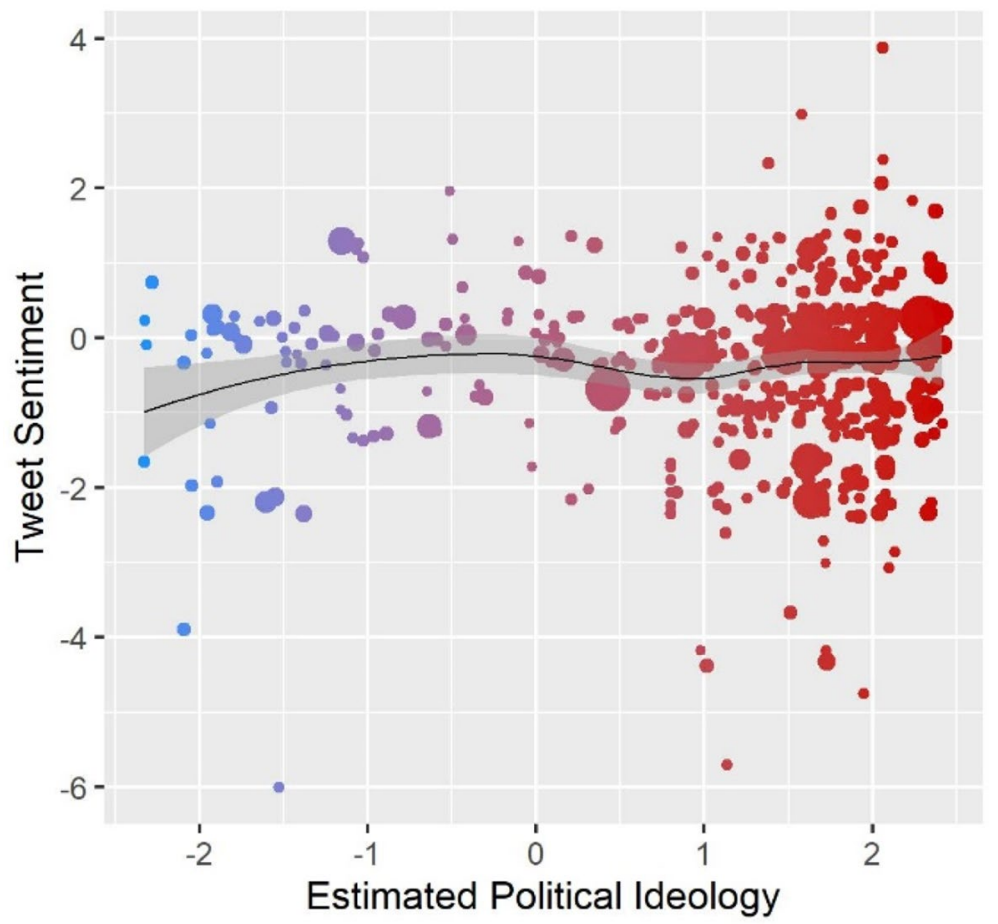

Fig. 6 Bill Gates Tweets

sense again. NEVER get a Chinese Virus vaccine especially from corrupt criminal Bill Gates." While there was not a clear relationship between tweet sentiment and political ideology with respect to tweets about Bill Gates, Twitter users with large followings are advancing unfounded conspiracy theories along clear partisan lines. In fact, half of Fox News viewers think Bill Gates is trying to surveil them with nasal cavity cameras implanted during the test for COVID-19. ${ }^{2}$ They even called for Department of Justice investigations into Bill Gates, among others, as user @ Smoke56895160 (2.4 estimated ideology) said: "AG Barr should investigate Dr Fauci and Dr Birx and Bill Gates and the WHO for their support and funding (taxpayer $\$$ ) of the Wuhan laboratory in communist China and the intentional manufacture and the release of the coronavirus resulting significant impact to millions of people."

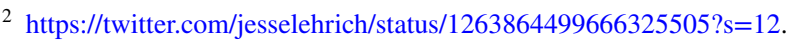




\section{CCPV Tweets}

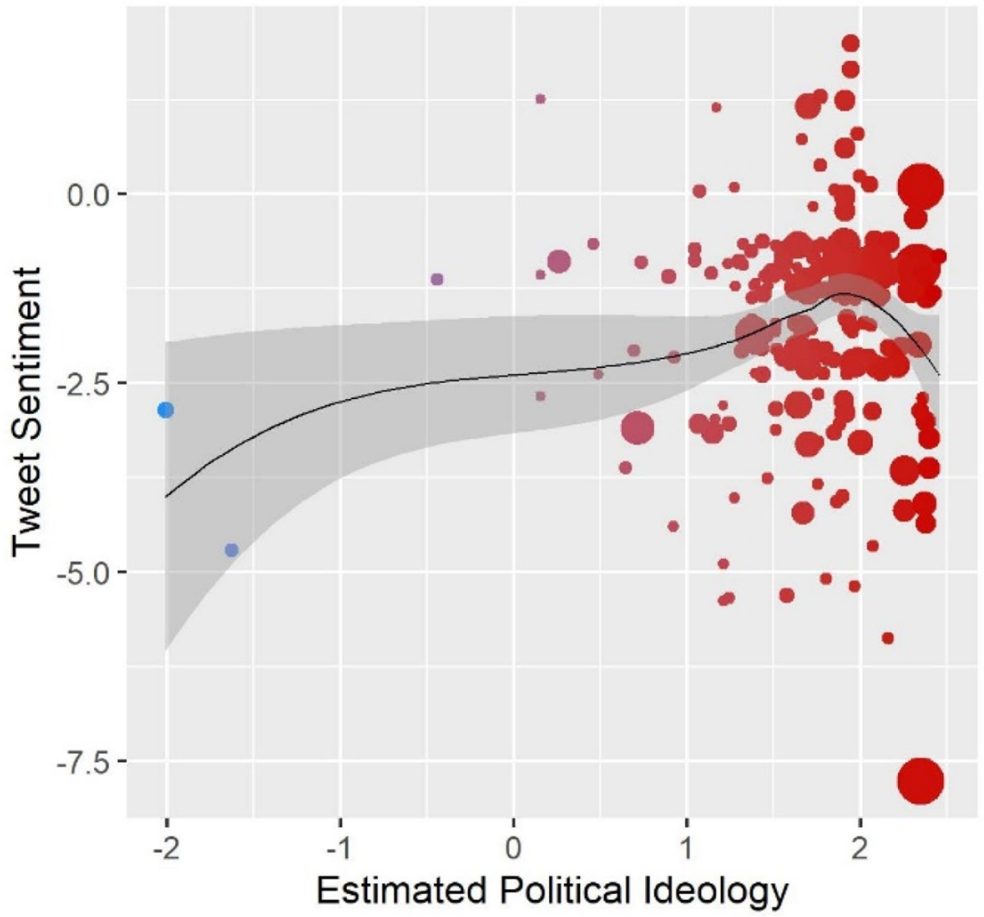

Fig. 7 CCPV Tweets

\section{Chinese communist party virus}

Similar to tweets about $5 \mathrm{G}$ and Bill Gates, I hypothesized $\left(\mathrm{H}_{3}\right)$ that tweets about the Chinese Communist Party creating COVID-19 intentionally would be more negative among conservative Twitter users. Figures 7 and 8 depict the relationship between tweet sentiment and political ideology for tweets about the "Chinese Communist Party Virus" and the "Deep State", respectively. With respect to tweets about the Chinese Communist Party Virus, the discourse was dominated and almost unilaterally conservative. Similarly, there is a clear negative trend associated with conservative political ideology and negative tweet sentiment regarding the topic, partially supporting $\mathrm{H}_{3}$. As the conspiracy theory that the Chinese Communist Party created a virus is almost exclusively conservative, an average negative sentiment on the topic tracks. As conservative Twitter users are almost unilaterally the only users of the term "Chinese Community Party Virus" or CCPV, tweets, like from user @Herbert_L_Reed (2.32 estimated ideology) "Being held in White House Rose Garden, @ realDonaldTrump just began the CCP Virus Task Force Briefing, if you're able to watch," range from descriptive to outwardly hostile, xenophobic and condemning of both China and the mainstream media (MSM): "I agree. And one 


\section{Deep State Tweets}

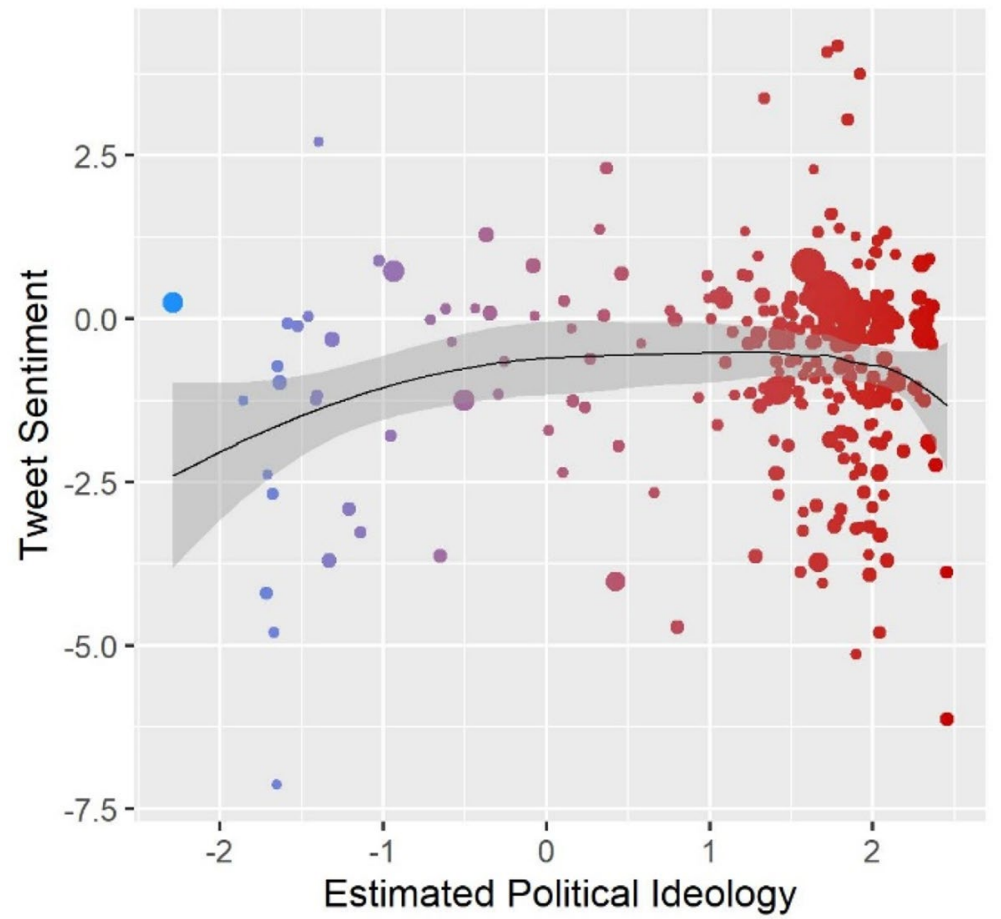

Fig. 8 Deep State Tweets

has to ask-which enemy is worse? The CV19/CCP Virus, or the fear-mongering, propaganda and lies of the MSM? The MSM/Deep State/Dems must be exposed and replaced! @Scavino45@toddeherman @dbongino@tracybeanz@JohnBWellsCTM @LindseyGrahamSC @ senatemajldr https://t.co/aF19ye7aRH” (User @geezermann, 2.16 estimated ideology; the tweet links to the president's tweet "FAKE NEWS! THE ENEMY OF THE PEOPLE!"). Like the earlier tweets about Bill Gates, these tweets specifically linked COVID-19 to China, the "mainstream media," Democrats, and the "Deep State," suggesting a clear conservative investment in each individual conspiracy theory and their connections on Twitter.

\section{Deep State}

I hypothesized $\left(\mathrm{H}_{3}\right)$ that tweets about the Deep State causing the COVID-19 pandemic would be increasingly negative for conservative Twitter users, particularly as the Deep State is a conspiracy theory unique to the conservative end of the political spectrum. Like the tweets about the CCPV, tweets about the Deep State were dominated by conservative Twitter users and there was a clear correlation between conservative ideology and negative tweet sentiment, partially supporting $\mathrm{H}_{3}$. In replies 
to journalists and political commentators criticizing the president's response to the COVID-19 pandemic, conservative Twitter users regularly assigned any dissidents with membership in the "Deep State". User @ MLG09217249 (2.03 estimated ideology) tweeted: “@CurtisHouck Olivia nuzzi is an ignorant deep state shill. How dumb. Pathetic to blame the President. The blame belongs on the backs of summer and piglousey and the demoncrats. They fight him every waking moment. The judgment of Almighty God is coming..." User @ NewRightAmerica (1.67 estimated ideology), criticizing the mainstream media and lauding conservatives, tweeted: “@ Hadrianus14@JoenormousM Sure, they deep state and the mainstream media told the lie that this is just another flu, bro. Then the terrible reality of the situation made that impossible. Only conservatives are willing to destroy their credibility with the flubro fantasty." Similarly, user @ AnthonyMichau18 (2.33 estimated ideology) tweeted that the Democrats and the Deep State were using the pandemic to tank the US economy to make the president look bad: " 2 deep state hacks that own this. Latest Numbers from Italy Prove Dr. Fauci and Dr. Birx Are Needlessly Destroying US Economy https://t.co/bmqXsiZqn8 via @ gatewaypundit”. Finally, like several users tweeting on other topics, user @ exavierpope ( -0.9 estimated ideology), criticizing conservative vloggers Diamond and Silk cut by Fox News for pushing conspiracy theories, tweeted "they [Diamond and Silk] also openly wondered whether 5G technology was being used to infect people with coronavirus in an effort to fill 'empty' hospitals, suggesting that the 'deep state snakes' were building towers in certain areas to "turn things on and off"'-Diamond \& Silk got tapped out https://t.co/zxJx3 UT8XE" and connected the conspiracy theories together.

\section{Discussion and conclusion}

Across five of six misinformation topics related to the COVID-19 pandemic (hydroxychloroquine, Bill Gates, 5G, CCPV, and the Deep State), conservatives dominated conversations on Twitter and openly criticized both their liberal counterparts and the "mainstream media" for criticizing the president's response to the pandemic and not seeing the true cause of the virus: Bill Gates, the Deep State, China, or 5G, depending on whose tweet you read. While $\mathrm{H}_{1}$ - that conservatives would dominate the discourse over misinformation topics-was only partially (5/6 topics) supported, it is clear that conservative Twitter users are driving the conversation around these misinformation topics and, consistent with previous research [20], more likely to condone ideologically consonant misinformation than their liberal peers. Similarly, $\mathrm{H}_{2}$ - that conservatives would be more supportive of the use of bleach and hydroxychloroquine for COVID-19 treatment-was refuted. While there was evidence of some support in the form of individual tweets, neutral and informative noise predominated the conversation on both topics. That being said, conservatives were still the primary discussants of hydroxychloroquine on Twitter, suggesting a willingness to engage in the spread of ideologically consonant misinformation. Finally, $\mathrm{H}_{3}$ - that conservatives would be more negative on the topics of 5G, Bill Gates, CCPV, and the Deep State-was partially supported and these 4 misinformation topics and conspiracy theories were clearly linked across conservative discussions. 
As other scholars have made clear, we know misinformation is happening, but how are we fighting it $[21,23]$ ? This study provided clear evidence that misinformation topics surrounding COVID-19 are being actively discussed on Twitter and that this discourse is predominantly weighted to the conservative end of the political spectrum and that conservative users are more active than their liberal counterparts $[51,52]$. As the analysis of previous pandemics has taught us, social media amplifies both disbelief in public health information and belief in associated misinformation topics and conspiracy theories, often to the result of reduced uptake of preventative measures and increased spread $[4,6]$. That the dangers of social media for public health are exacerbated by political polarization is of even further concern, particularly because conservatives are already more likely to condone misinformation from their leadership, accept information from their leadership as high quality, and criticize opposing information [1, 14-16, 51, 52]. Within the context of the current COVID-19 pandemic, Fox News viewers (conservatives) have already been shown to comply with a stay at home order substantially less than their liberal peers and $1 / 3$ of respondents to a CDC study have been incorrectly (including injection and ingestion) using bleach as a preventative measure for COVID-19. ${ }^{3-4}$ Whereas Democrats are more likely to criticize the government's response and discuss testing and increased public health measures, Republicans are more likely to blame China, discuss the impact on the economy, accusing the mainstream media of lying, and engage in panic buying of consumer goods $[51,52] .^{5}$

\section{Implications and future research}

What does this mean for public health? Though COVID-19 is deadliest in more liberal areas, largely due to structural issues like poverty, healthcare disparities, and housing density, we may see spikes in more conservative areas, particularly as cities and states headed by conservative politicians ignore the advice of public health experts and reopen. ${ }^{6}$ How can we combat the negative impacts of misinformation amplified by social media? First, Twitter could consider suspending accounts credibly identified as engaging in misinformation. Though this has not consistently happened in the past (and Twitter has been routinely criticized by conservatives for "censoring" their voices and has subsequently acquiesced, likely due to the adrevenue incentive to keep conservatives on the platform), monitoring of sensitive topics could mitigate the amplification of misinformation that is causing people to reject public health information [53]. Similarly, Twitter could remove potentially harmful topics from Twitter discourse without suspending accounts. While my immediate recommendations focus on what Twitter, as a platform, could do to stop

\footnotetext{
3 https://twitter.com/mattgrossmann/status/1264881959639166976?s=12.

4 https://www.cdc.gov/mmwr/volumes/69/wr/mm6923e2.htm?s_cid=mm6923e2_w.

5 https://medium.com/@KerleIndia/ideological-polarisation-and-pandemics-structural-topic-modellingof-online-covid-19-discourse-2b72b92320d5.

6 https://www.nytimes.com/2020/05/25/us/politics/coronavirus-red-blue-states.html.
} 
the spread of misinformation, greater education on source assessment and plausibility may assist the average person in parsing what is real and what is \#FakeNews [49]. Similarly, further research on the spread of misinformation, particularly as it relates to concerning public topics like a pandemic, should focus on cluster analysis and information flows. While this study identified users with large followings engaging in misinformation or simply discussion about a misinformation topic, I did not investigate how wide these accounts reach or how diluted (or amplified) their messaging becomes as it travels from one Twitter user to the next [see 51 and 52 for more detailed network analyses]. Finally, further research is needed that more cleanly parses Twitter data associated with misinformation topics. While sentiment analysis is an expedient way to process large corpuses of data, it is often unable to identify instances of sarcasm, satire, or separate positively intoned language from an objective statement.

Funding There is no funding associated with this manuscript.

Code availability All code available by request to author.

\section{Compliance with ethical standards}

Conflicts of interest No relevant disclosures.

Availability of data and material All data and analysis code available by request to author.

\section{References}

1. Nisbet, E. C., Cooper, K. E., \& Garrett, R. K. (2015). The partisan brain: How dissonant science messages lead conservatives and liberals to (dis) trust science. The ANNALS of the American Academy of Political and Social Science, 658(1), 36-66.

2. Ahmed, W., Vidal-Alaball, J., Downing, J., \& Seguí, F.L. (2020). Dangerous messages or satire? analysing the conspiracy theory linking $5 \mathrm{~g}$ to covid-19 through social network analysis. J. Med Internet Res.

3. Shahsavari, S., Holur, P., Tangherlini, T. R., \& Roychowdhury, V. (2020). Conspiracy in the time of Corona: Automatic detection of Covid-19 conspiracy theories in social media and the news. arXiv preprint. arXiv:2004.13783.

4. Chew, C., \& Eysenbach, G. (2010). Pandemics in the age of Twitter: content analysis of Tweets during the 2009 H1N1 outbreak. PLoS ONE, 5(11), e14118.

5. Constantinou, M., Kagialis, A. \& Karekla, M., (2020). Is science failing to pass its message to people? Reasons and risks behind conspiracy theories and myths regarding COVID-19. Reasons and risks behind conspiracy theories and myths regarding COVID-19 (April 16, 2020).

6. McNeill, A., Harris, P. R., \& Briggs, P. (2016). Twitter influence on UK vaccination and antiviral uptake during the $2009 \mathrm{H} 1 \mathrm{~N} 1$ pandemic. Frontiers in public health, 4, 26.

7. Bimber, B., \& Gil de Zúñiga, H. (2020). The unedited public sphere. New Media \& Society, 22(4), $700-715$.

8. Bright, J. (2018). Explaining the emergence of political fragmentation on social media: The role of ideology and extremism. Journal of Computer-Mediated Communication, 23(1), 17-33.

9. Colleoni, E., Rozza, A., \& Arvidsson, A. (2014). Echo chamber or public sphere? Predicting political orientation and measuring political homophily in Twitter using big data. Journal of Communication, 64(2), 317-332. 
10. Barberá, P., Jost, J. T., Nagler, J., Tucker, J. A., \& Bonneau, R. (2015). Tweeting from left to right: Is online political communication more than an echo chamber? Psychological Science, 26(10), 1531-1542.

11. Himelboim, I., McCreery, S., \& Smith, M. (2013). Birds of a feather tweet together: Integrating network and content analyses to examine cross-ideology exposure on Twitter. Journal of ComputerMediated Communication, 18(2), 154-174.

12. Gil de Zúñiga, H., Koc Michalska, K., \& Römmele, A. (2020). Populism in the era of Twitter: How social media contextualized new insights into an old phenomenon. New Media \& Society, 22(4), 585-594.

13. Ernst, N., Engesser, S., Büchel, F., Blassnig, S., \& Esser, F. (2017). Extreme parties and populism: An analysis of Facebook and Twitter across six countries. Information, Communication \& Society, 20(9), 1347-1364.

14. Hahl, O., Kim, M., \& Zuckerman Sivan, E. W. (2018). The authentic appeal of the lying demagogue: Proclaiming the deeper truth about political illegitimacy. American Sociological Review, 83(1), 1-33.

15. Swire, B., Berinsky, A. J., Lewandowsky, S., \& Ecker, U. K. (2017). Processing political misinformation: comprehending the Trump phenomenon. Royal Society Open Science, 4(3), 160802.

16. Wells, C., Shah, D., Lukito, J., Pelled, A., Pevehouse, J. C., \& Yang, J. (2020). Trump, Twitter, and news media responsiveness: A media systems approach. New Media \& Society, 22(4), 659-682.

17. Zerback, T., Töpfl, F., \& Knöpfle, M. (2020). The disconcerting potential of online disinformation: Persuasive effects of astroturfing comments and three strategies for inoculation against them. New Media \& Society, 1461444820908530.

18. Lewandowsky, S., Ecker, U. K., \& Cook, J. (2017). Beyond misinformation: Understanding and coping with the "post-truth" era. Journal of Applied Research in Memory and Cognition, 6(4), 353-369.

19. Ribeiro, M. H., Calais, P. H., Almeida, V. A., \& Meira Jr, W. (2017). " Everything I disagree with is \#fakenews": Correlating political polarization and spread of misinformation. arXiv preprint arXiv: 1706.05924 .

20. Roets, A. (2019). Is there an ideological asymmetry in the moral approval of spreading misinformation by politicians? Personality and Individual Differences, 143, 165-169.

21. Weeks, B. E., Kim, D. H., Hahn, L. B., Diehl, T. H., \& Kwak, N. (2019). Hostile media perceptions in the age of social media: Following politicians, emotions, and perceptions of media bias. Journal of Broadcasting \& Electronic Media, 63(3), 374-392.

22. Kahne, J., \& Bowyer, B. (2017). Educating for democracy in a partisan age: Confronting the challenges of motivated reasoning and misinformation. American Educational Research Journal, 54(1), 3-34.

23. Weeks, B. E., \& Gil de Zúñiga, H. (2019). What's next six? Observations for the future of political misinformation research. American Behavioral Scientist. https://doi.org/10.1177/000276421987823 6

24. Bail, C. A. (2014). The cultural environment: Measuring culture with big data. Theory and Society, 43(3-4), 465-482.

25. Steinert-Threlkeld, Z. C. (2018). Twitter as Data. Cambrindge: Cambridge University Press.

26. Christensen, D., \& Garfias, F. (2018). Can you hear me now? How communication technology affects protest and repression. Quarterly Journal of Political Science, 13(1), 89.

27. Freelon, D., McIlwain, C. D., \& Clark, M. (2016). Beyond the hashtags: \# Ferguson, \# Blacklivesmatter, and the online struggle for offline justice. Center for Media \& Social Impact, American University, Forthcoming.

28. Little, A. T. (2016). Communication technology and protest. The Journal of Politics, 78(1), 152-166.

29. Steinert-Threlkeld, Z. C. (2017). Spontaneous collective action: Peripheral mobilization during the Arab Spring. American Political Science Review, 111(2), 379-403.

30. Blevins, J. L., Lee, J. J., McCabe, E. E., \& Edgerton, E. (2019). Tweeting for social justice in \#Ferguson: Affective discourse in Twitter hashtags. New Media \& Society, 21(7), 1636-1653.

31. Modrek, S., \& Chakalov, B. (2019). The\# MeToo movement in the United States: text analysis of early twitter conversations. Journal of Medical Internet Research, 21(9), e13837.

32. Saxton, G. D., Niyirora, J., Guo, C., \& Waters, R. (2015). \# AdvocatingForChange: The strategic use of hashtags in social media advocacy. Advances in Social Work, 16(1), 154-169. 
33. Abdelrazeq, A., Janßen, D., Tummel, C., Jeschke, S., \& Richert, A. (2016). Sentiment analysis of social media for evaluating universities. In Automation, Communication and Cybernetics in Science and Engineering (pp. 233-251). Springer, Cham.

34. Abeywardena, I. S. (2014). Public opinion on OER and MOOC: A sentiment analysis of twitter data. In International Conference on Open and Flexible Education (pp. 296-307).

35. Hixson, T. (2020). Reactions vs. reality: Using sentiment analysis to measure university students' responses to learning ArcGIS. Journal of Map \& Geography Libraries, 1-14.

36. Saif, H., He, Y., \& Alani, H. (2012). Semantic sentiment analysis of twitter. In International Semantic Web Conference (pp. 508-524). Springer, Berlin.

37. Attri, I., \& Dutta, M. (2020). Review of various sentiment analysis approaches. In Proceedings of International Conference on IoT Inclusive Life (ICIIL 2019), NITTTR Chandigarh, India (pp. 223234). Springer, Singapore.

38. Jena, R. K. (2019). Sentiment mining in a collaborative learning environment: capitalising on big data. Behaviour \& Information Technology, 38(9), 986-1001.

39. Kumar, A., \& Sebastian, T. M. (2012). Sentiment analysis on twitter. International Journal of Computer Science Issues (IJCSI), 9(4), 372.

40. Kumar, A., \& Jaiswal, A. (2020). Systematic literature review of sentiment analysis on Twitter using soft computing techniques. Concurrency and Computation: Practice and Experience, 32(1), e5107.

41. Pak, A., \& Paroubek, P. (2010, May). Twitter as a corpus for sentiment analysis and opinion mining. In LREc (Vol. 10, No. 2010, pp. 1320-1326).

42. Abdulsalami, A. O., Ahmad, B. I., Umar, M. A., Abubakar, A. H., Jauro, F., Kufena, A. M., \& Ekoja, E. A. (2017, November). Sentiment analysis of students' perception on the use of smartphones: A cross sectional study. In 2017 Second International Conference on Informatics and Computing (ICIC) (pp. 1-5). IEEE.

43. Zhang, L., Ghosh, R., Dekhil, M., Hsu, M., \& Liu, B. (2011). Combining lexicon-based and learning-based methods for Twitter sentiment analysis. HP Laboratories, Technical Report HPL-2011, 89.

44. Liu, B. (2012). Sentiment analysis and opinion mining: synthesis lectures on human language technologies \# 16. San Rafael: Morgan and Claypool Publishers.

45. Jain, S., Sharma, V., \& Kaushal, R. (2016, September). Towards automated real-time detection of misinformation on Twitter. In 2016 International Conference on Advances in Computing, Communications and Informatics (ICACCI) (pp. 2015-2020). IEEE.

46. Kušen, E., \& Strembeck, M. (2018). Politics, sentiments, and misinformation: An analysis of the Twitter discussion on the 2016 Austrian Presidential Elections. Online Social Networks and Media, 5, 37-50.

47. Zollo, F., Novak, P. K., Del Vicario, M., Bessi, A., Mozetič, I., Scala, A., Caldarelli, G. \& Quattrociocchi, W. (2015). Emotional dynamics in the age of misinformation. PloS one, 10(9), p.e0138740.

48. Barberá, P. (2015). Birds of the same feather tweet together: Bayesian ideal point estimation using Twitter data. Political Analysis, 23(1), 76-91.

49. Sinatra, G. M., \& Lombardi, D. (2020). Evaluating sources of scientific evidence and claims in the post-truth era may require reappraising plausibility judgments. Educational Psychologist, 1-12.

50. Liu, B., Hu, M., \& Cheng, J. (2005, May). Opinion observer: analyzing and comparing opinions on the web. In Proceedings of the 14th International Conference on World Wide Web (pp. 342-351).

51. Smith, M., McAweeney, E., \& Ronzaud, L. (2020). The COVID-19 "Infodemic". Technical report, Apr. 2020. Library Catalog: graphika.com.

52. Graham, T., Bruns, A., Zhu, G., \& Campbell, R. (2020). Like a virus: The coordinated spread of Coronavirus disinformation.

53. Noble, S. U. (2018). Algorithms of Oppression: How Search Engines Reinforce Racism. New York: NYU Press.

Publisher's Note Springer Nature remains neutral with regard to jurisdictional claims in published maps and institutional affiliations. 